tätskontrolle nationaler Parlamente“ (S. 461 f. und S. 469 - 472) erarbeitet; denn auf die Effizienz dieser innerstaatlichen Abläufe kommt es entscheidend an. Dass es an dieser Effizienz noch vieles zu verbessern gibt, hat schon die Untersuchung von Janowski in einem noch weiter gespannten Rahmen ergeben. Die von Mellein daraus gezogenen Folgerungen sind deshalb auch sehr beachtenswert.

Das gleicht aber nicht die Mängel aus, die aus den nicht hinreichend oder gar nicht gegebenen Antworten zu den Fragen einer direkten Einfügung nationaler parlamentarischer Willensbildung in die EU-Strukturen durch das Frühwarnsystem resultieren. Die Konfliktsituationen, die sich aus diesem System faktisch wie rechtlich ergeben, werden entweder gar nicht gesehen oder durch Euphorie über das Neue verdeckt. So sieht die Verfasserin die „direkte Einbeziehung der Parlamente im Anfangsstadium des Gesetzgebungsprozesses (als) eine neuartige ... Quelle demokratischer Legitimation“ an; denn es sei „ein Novum, dass dasjenige Organ, welches den Gesetzgebungsvorschlag vorlegt, der unmittelbaren Kontrolle aller mitgliedstaatlichen Parlamente unterliegt" (S.491). So weit, so gut - aber ist diese Neuartigkeit allein schon eine hinreichende Rechtfertigung für die durch sie heraufbeschworenen Vermischungsprobleme? Ohne jene Probleme auch nur annähernd hinreichend zu erörtern, konstatiert Mellein dazu schlicht, durch jene direkte Einbeziehung werde „die Beratung über Art, Sinn und Zweck der Gesetzgebung auf EU-Ebene ... verbessert, ohne dass die Entscheidungsfindung der Union wesentlich verzögert und das institutionelle Gleichgewicht gestört würden“" (S. 493). Nähere Erörterungen und Beweise für beides bleibt sie allerdings schuldig, und darin liegt der wesentliche Mangel des Buches.

Beide Untersuchungen sind zweifellos konkret für die noch ausstehende Praxis besonders nützlich. Der von Janowski gebührt aber der Vorrang, weil sie tiefer in die von der Einbindung nationaler Parlamente in die EU-Rechtsetzung heraufbeschworenen verfassungsrechtlichen Probleme eindringt, die von Mellein ignoriert oder zumindest verdeckt werden.

Uwe Leonardy

Der Kommissionspräsident der EU: Staatsoberhaupt, Regierungschef, „europäische Leitfigur"?

Staeglich, Simone: Der Kommissionspräsident als Oberhaupt der Europäischen Union. Vom primus inter pares zur europäischen Leitfigur, Duncker \& Humblot, Berlin 2007, 294 Seiten, $€ 72,-$.

Die Wahlen zum Europäischen Parlament zeichnen sich bisher nicht nur durch eine niedrige Wahlbeteiligung aus, sondern werden auch überwiegend mit Themen der nationalen Politik bestritten. In Deutschland gelten sie ähnlich wie Landtagswahlen als Stimmungsbarometer für die Unterstützung der Bundestagsparteien in der Bevölkerung. Als Gründe hierfür nennt man die fehlende Transparenz der Entscheidungswege in der EU und die fehlende Personalisierung der europäischen Politik. Während in den Mitgliedstaaten eine Wahl gleichzeitig als eine Entscheidung für oder gegen Tony Blair, Nicolas Sarkozy, Silvio Berlusconi oder Gerhard Schröder aufgefasst wird, fehlt der europäischen Politik das im Fernsehzeitalter so wichtige Gesicht. 
Simone Staeglich untersucht deshalb in ihrer Studie, ob der Präsident der EU-Kommission diese Lücke ausfüllen und eine „europäische Leitfigur“" werden kann. Sie beschreibt die rechtlichen und politischen Kompetenzen des Präsidenten ausführlich in ihrer historischen Entwicklung und zeigt, wie weitgehend dieser sich inzwischen einem Regierungschef angenähert hat: Seit Walter Hallstein, dem ersten Kommissionspräsidenten von 1958 bis 1967 , wuchs der Einfluss des Amtes kontinuierlich. Gleichzeitig änderte sich seine Legitimationsbasis und das Selbstverständnis seiner Inhaber: Während Politiker wie Charles de Gaulle und Margret Thatcher die Kommission als verwaltendes Sekretariat betrachteten, verstanden sich ihre Präsidenten seit Gaston Thorn (1981 bis 1985) und Jacques Delors (1985 bis 1995) als Leiter einer europäischen Regierung, die allerdings durch die Unzulänglichkeit der sie umgebenden Institutionen behindert wurde. Heute verfügt der Präsident der Kommission zum Beispiel über eine Richtlinienkompetenz, die in der EU in Anlehnung an den Vertrag von Nizza als „Leitlinienkompetenz“ bezeichnet wird. Seit 2001 hat er außerdem die Möglichkeit, den Rücktritt von Kommissionsmitgliedern durchzusetzen. Die Autorin vertritt zu Recht die These, dass die inzwischen vorgeschriebene Zustimmung des Europäischen Parlaments zur Ernennung des Präsidenten dessen Position gestärkt hat. Der amtierende Präsident José Manuel Barroso musste allerdings auf Drängen des Parlaments ein Kommissionsmitglied austauschen (Rocco Buttiglione) und mehreren Kommissaren andere Aufgaben als vorgesehen zuweisen.

Die Arbeit von Staeglich ist eine rechtswissenschaftliche Dissertation und stützt sich überwiegend auf Verträge und andere Rechtsakte der Union. Politische Ereignisse werden von Fall zu Fall als Erläuterung beigefügt. Das Scheitern der Kommission von Jacques Santer oder die Probleme bei der Bildung der Kommission Barroso im Jahre 2004 werden zwar erwähnt, aber nicht hinsichtlich ihrer politischen Wirkung analysiert. Die Arbeit überzeugt durch eine übersichtliche Gliederung und durch eine umfassende Berücksichtigung der Dokumente sowie der juristischen Literatur. Die Autorin untersucht die Entwicklung bis zum gescheiterten Verfassungsvertrag. Die Einigung auf den neuen Verfassungskompromiss im Vertrag von Lissabon konnte nicht mehr berücksichtigt werden. Da dessen Ratifizierung in den 27 Staaten der Union gerade erst begonnen hat, endet die Studie in einer OpenEnd-Situation.

Ein wenig irritierend ist, dass Staeglich den Kommissionspräsidenten der EU mit den Präsidenten Deutschlands und Frankreichs vergleicht. Die Position dieser beiden Staatsoberhäupter beschreibt die Autorin zu Beginn ihres Buches in einer geglückten Zusammenfassung. Dabei wird aber deutlich, dass der deutsche Bundespräsident für einen sinnvollen Vergleich mit dem Kommissionspräsidenten kaum ausreichende Befugnisse hat. Der politische Einfluss seines französischen Kollegen wechselt mit der parteipolitischen Konstellation. Unter den Bedingungen der Kohabitation wird von ihm ein anderes Verhalten erwartet als beim politischen Gleichklang mit der Parlamentsmehrheit. Seine Amtsführung ist deshalb überwiegend von Konventionen bestimmt, die sich einer staatsrechtlichen Einordnung entziehen. Es überrascht daher nicht, wenn die Autorin im Schlussteil den Kommissionspräsidenten auch mit dem „Regierungschef eines Staates, der vom Parlament abhängig ist" vergleicht (S. 223). Er hat im politischen System der EU demnach bereits eine ähnliche Position wie der deutsche Bundeskanzler und der französische Premierminister zur Zeit der Kohabitation (S. 237). Simone Staeglich hätte deshalb von vornherein den Vergleich mit den Premierministern in parlamentarischen Regierungssystemen ins Auge fassen müssen. Der bis auf Hans Kelsen zurückverfolgte Begriff des „Oberhaupts“ aus der Allgemeinen 
Staatslehre wies sie allerdings in eine andere Richtung. Für den Politikwissenschaftler entsteht deshalb der Eindruck, die Rahmenhandlung stimme mit dem überzeugend dargestellten Hauptthema nicht überein.

Der Präsident der Kommission kann durchaus zur europäischen Leitfigur werden - aber nur in seiner Eigenschaft als Regierungschef. Nach dem neuen EU-Vertrag steht neben ihm (oder über ihm) der erstmals für einen Zeitraum von zweieinhalb Jahren zu wählende Präsident des Europäischen Rates.

Karlheinz Niclauß

\section{Parlamentarische Untersuchungsausschüsse in den USA und in Deutschland: enttäuschender Rechtsvergleich}

Bräcklein, Susann: Investigativer Parlamentarismus. Parlamentarische Untersuchungen in der Bundesrepublik Deutschland und den Vereinigten Staaten von Amerika, Verlag Duncker \& Humblot (Beiträge zum Parlamentsrecht, Band 62), Berlin 2006 (zugleich: Dissertation, Humboldt Universität Berlin 2004), 394 Seiten, € 76,-.

Mit der Verabschiedung des Gesetzes zur Regelung des Rechts der Untersuchungsausschüsse (PUAG) schloss der Bundestag 2001 nach jahrzehntelanger gesetzgeberischer Untätigkeit eine ausgesprochen ärgerliche Lücke in seinem Parlamentsrecht. Es ist vor allem den erfahrenen Parlamentariern Erika Simm (SPD), Hermann Bachmair (SPD), Andreas Schmidt (CDU/CSU) und Hans-Christian Ströbele (Bündnis 90/Die Grünen) zu verdanken, dass ein ausgewogenes Gesetz zustande kam, das einstimmig vom Bundestag angenommen wurde. Auch in der Rückschau bleibt bemerkenswert, dass die damalige rot-grüne Parlamentsmehrheit bereit war, durch die besondere Ausformung von Minderheitsrechten im PUAG die Macht der Parlamentsmehrheit zugunsten der Minderheit zu begrenzen. Es wundert nicht, dass sich inzwischen mehrere Dissertationen dem wichtigen PUAG widmen.

Eine insgesamt enttäuschende Arbeit mit wenig überzeugenden Thesen legt Susann Bräcklein vor. Bereits der Titel ist prätentiös, und dem selbst gesetzten Anspruch wird die Autorin auf den 394 Seiten ihrer Arbeit selten gerecht. Die Monographie ist rechtsvergleichend angelegt und leidet spürbar an dem Mangel vieler rechtsvergleichender Arbeiten: Weder das nationale Recht noch das fremde Recht werden angemessen durchdrungen. Die Schlussfolgerungen, die Bräcklein aus dem Rechtsvergleich zieht, sind zudem problematisch.

Die Autorin referiert (S. 53 - 111) das Untersuchungsrecht des Bundestages, ohne auch nur an einer einzigen Stelle zu neuen Erkenntnissen zu kommen. Die Darstellung des Untersuchungsrechts des US-Kongresses (S. 112 - 230) hat ebenfalls eher summarischen, deskriptiven Charakter. Nach US-amerikanischem Verfassungsverständnis wird das Untersuchungsrecht mit der „implied powers“-Theorie begründet. Diese schließt von der Aufgabe auf die Befugnisse. Die auch mit Zwangsmitteln ausgestatteten Untersuchungsbefugnisse ergeben sich danach aus den Gesetzgebungs- und Kontrollkompetenzen des US-Kongresses.

Der dritte Teil des Buches („Vergleichende Analyse und Reformüberlegungen“, S. 231 - 372) bildet den problematischen Schwerpunkt der Arbeit. Ausgehend von der „implied powers“-Theorie meint die Autorin, Art. 44 GG sei keine Ausnahmeregelung zugunsten 